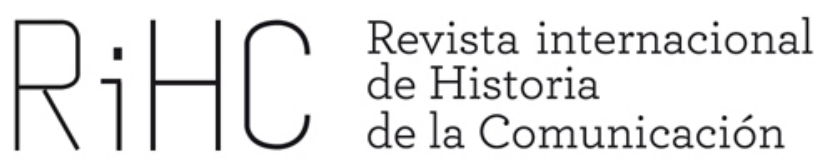

\title{
CATALUÑA, NACIÓN Y MEDIOS. LA CONSTRUCCIÓN DEL ESPACIO NACIONAL DE COMUNICACIÓN (1978-2014) $)^{1}$
}

DOI: http://dx.doi.org/10.12795/RiHC.2014.i03.06

Pablo Giori

Universidad de Girona

pablogiori@gmail.com

Recibido: 6-9-2014

Aceptado: 14-11-2014

Resumen: Crear el espacio nacional de comunicación era una necesidad práctica que se convierte en un concepto teórico desarrollado por la Escuela catalana de comunicación a comienzos de los años 70. Este propone a las nacientes instituciones políticas catalanas, recuperadas con la transición, un compromiso por crear unos medios catalanes de comunicación capaces de reproducir y socializar en la nueva nación. El artículo recupera esta propuesta, su evolución y sus principales actores, haciendo un balance sobre la situación en los diferentes momentos históricos para comprender la situación en la actualidad.

\footnotetext{
${ }^{1}$ Esta investigación recibe el soporte de una beca FPU del Ministerio de Educación, Cultura y Deporte (MECD) del Gobierno de España.
} 
Palabras clave: Cataluña, nacionalismo, espacio nacional de comunicación, Josep Gifreu, medios de comunicación.

\begin{abstract}
The creation of a national space of communication was a practical need that becomes a theoretical concept developed by the Catalan School of Communication in the 70s. This concept proposes to the newly recovered Catalan political institutions a major commitment to create a Catalan mass media system powerful enough to reproduce and to socialize in the new Catalan nation. This article recovers this proposal, its evolution and its major agents to provide a historical balance of the situation untill the present days.
\end{abstract}

Keywords: Catalonia, nationalism, national space of comunication, Josep Gifreu, mass media.

\title{
Introducción
}

Este artículo pretende repasar la historia de una serie de teorías que rondan la problemática propuesta por el concepto de "construcción del espacio nacional de comunicación", a partir de las reflexiones de Josep Gifreu, Miquel de Moragas y Joan Manuel Tresserras. Dentro de un contexto de lo que algunos llaman de "reconstrucción nacional", la Escuela Catalana de Comunicación comienza a reflexionar sobre el papel de los medios, y de la universidad misma, en la construcción de una Cataluña postfranquista.

A partir del regreso de la democracia y de las primeras elecciones autonómicas que dan como victorioso a un partido nacionalista moderado, Convergencia i Unió (CiU), la universidad catalana y el campo académico de estudios sobre comunicación comienza lentamente a configurarse, en un diálogo permanente entre reflexión teórica y práctica mediática. Es también el contexto de las reflexiones sobre el Nuevo Orden Mundial de la Comunicación y con el Congreso de Cultura Catalana de 1978 algunos investigadores catalanes se plantean la necesidad de llevar adelante una investigación teórica, evaluativa, práctica y política, sobre la necesidad de construir unas instituciones mediáticas propias para una Cataluña, y unos Países Catalanes ${ }^{2}$, que consideran una nación diferente de la española (de Moragas, 1988: 39 y Gifreu, 1986b). Así es que la necesidad de erigir este espacio nacional de comunicación nace con tres objetivos: construir medios de socialización para una "nueva Cataluña", fortalecer el campo

\footnotetext{
${ }^{2}$ El concepto de Países Catalanes abarca, para los autores, aquello que une a los territorios de habla y de cultura catalana: Valencia, Baleares, Cataluña y la Cataluña Nord en Francia (Gifreu, 1991: 23). Ver también Romeu Jover (1977).
} 
catalán de comunicación y producir nuevas instancias de acción práctica para los intelectuales. En este sentido sostenían que construir este espacio de diálogo que abarcara las comunidades de lengua y cultura catalanas era también reconstruir la nación catalana, Cataluña, pero también fundar un campo de estudios académicos sobre comunicación.

El artículo se organiza en tres partes: la primera es una introducción con aportes metodológicos; la segunda entra en tema con unas propuestas teóricas, el contexto histórico en que se desarrollan las mismas y un recorrido histórico por los aportes teóricos más importantes para concluir con un balance; y una tercera parte donde se esbozan las conclusiones. La propuesta, entonces, hace una historia que busca ayudarnos a pensar en la relación existente entre los medios masivos de comunicación y la construcción de colectividad; sobre la nación, la identidad nacional y los medios y sobre el papel de estos en la socialización (la interiorización de la nación) y el nation building.

\section{Metodología}

El trabajo se estructuró sobre dos momentos, superpuestos en los resultados. Para el primer momento desarrollé una recogida de datos de fuentes secundarias, los libros y artículos académicos e institucionales de los autores centrales, que son analizados y sintetizados en una discusión histórica general. En un segundo momento, desarrollé entrevistas en profundidad con Josep Gifreu (en febrero de 2013), centrales para enriquecer un balance historiográfico, y una lectura y corrección del texto final para puntualizar algunos aspectos. La selección de Josep Gifreu como el entrevistado clave proviene de su continuidad en la reflexión sobre la temática, una que recorre toda su producción académica y política. En el caso de los otros dos autores trabajados, Miquel de Moragas y Joan Manuel Tresserras, estos han ido cambiando de tema de investigación o han desarrollado la mayor parte de su trabajo dentro de la política partidista, lo que se aleja del tema aquí expuesto.

El arco temporal, 1975 y 2014, es el resultado del marco temporal que los propios autores marcan como centrales para su tarea investigadora y que se abre con una ventana de oportunidad con la llegada de la democracia y se cierra con una etapa que consideran de declive (Gifreu, 2012). A partir de los sucesos políticos de 2012 en relación a la posible independencia de Cataluña, se renuevan las expectativas sobre el "espacio nacional de comunicación". Recuperar los discursos de forma indirecta, los textos producidos para difundir las ideas en su momento inicial, y ponerlos en diálogo 
con las entrevistas desde las fuentes primarias, nos permitió completar el arco temporal de toda la gestación y desarrollo de la propuesta analizada.

\section{Construir el espacio nacional de comunicación}

Entendidos como los principales generadores de representaciones sociales y culturales en las sociedades contemporáneas, los medios hace tiempo que han conseguido superar a instituciones tradicionales como la escuela o la familia en la constitución de un imaginario colectivo. (Castelló, 2007: 62).

\subsection{Marco y propuestas teóricas}

La relación existente entre los medios masivos y la construcción de colectividad, desde su capacidad de crear agendas compartidas, cartografías y referentes hasta su capacidad de representación y de construir realidad, ha ido creciendo a medida que estos han ido tomando un papel más importante en la vida cotidiana. En el periodo comprendido por este trabajo, 1975 y 2014, los medios masivos, las nuevas tecnologías y los social media, han sido motores del cambio en las formas de socialización. Hoy los medios ocupan un lugar central en la forma en que construimos nuestra identidad y su lógica, la media logic, nos han enseñado a comunicar y a convivir de una manera totalmente nueva. Dentro de este contexto, Cataluña desarrolló varias estrategias paralelas para apropiarse de las instituciones que le permitirían reconstruirse, y reconsiderarse, como una nación: las instituciones políticas, los centros educativos, la sociedad civil y los medios de comunicación.

Son muchos los autores que han teorizado sobre la nación, la identidad nacional y los medios. Como lo explica Àlvar Peris Blanes (2008: 4), en su afán por determinar las causas que contribuyeron a la construcción y consolidación de la nación durante los siglos XVIII y XIX, los teóricos modernistas han destacado la importancia del sistema comunicativo. Sin duda fue Karl Deutsch con su libro Nationalism and Social Communication de 1966 quien puso más énfasis en la participación de la estructura comunicativa en este proceso, íntimamente ligada al desarrollo industrial y tecnológico. Pero fue Ernest Gellner en Nations and Nationalism de 1983 quien constató la trascendencia de los medios masivos en la formación de las "comunidades 
imaginadas" y del Estado-nación y especialmente cuando éste quiere incorporar a sectores más amplios de población.

Como señala desde una perspectiva histórica Josu Amezaga (2004: 2), así como no se puede dudar sobre la importancia que tuvo la imprenta en la formación de la identidad nacional hacia mediados del siglo XIX, tampoco puede haber dudas sobre la importancia que han tenido la radio y la televisión para la homogeneización cultural de los Estados nación durante el siglo XX. En este proceso, algunas de las comunidades culturales han conseguido hacer llegar su propia lengua a las nuevas instituciones y otras han tenido que resignar este aspecto fundamental de su identidad.

De hecho, los Estados-nación han puesto siempre mucho esmero en impulsar y regular toda una serie de infraestructuras que permitan la efectividad de unas políticas de cultura y comunicación en la consolidación de su legitimidad política y cultural (de Moragas, 1988: 29).

Por la misma línea va lo propuesto por Enric Castelló cuando habla de la importancia de la creación de sistemas mediáticos nacionales, al mismo tiempo que nos recuerda las medidas proteccionistas implementadas en diversos países para defenderse frente a la entrada masiva de productos provenientes de las industrias culturales norteamericanas.

Si tienes un Estado, las políticas son aplicadas naturalmente, automáticamente. Todo el mundo implementa políticas culturales. El Estado implementa sus políticas como si fuese esta su misión primordial. Todo el mundo lo da por sentado. Pero si no tienes un Estado, incluso te sientes como pidiendo perdón o, por lo menos, como justificándote (Castelló, 2012: 99).

Los medios masivos son fundamentales para consolidar la democracia y para fundar una memoria colectiva que proponga puntos de referencia compartidos, una nación.

Como indicamos en otra investigación, hay casos de Estados donde existe una coordinación perfecta entre el Estado y la nación representada, donde no hay un conflicto, y hay otros que son Estados plurinacionales, donde esta relación es conflictiva (Giori, 2013: 808). En estos casos, suele producirse un conflicto cuando los Estados, controlados principalmente por una de las varias naciones en sus fronteras, se autorepresentan como uninacionales, dejando de lado la pluralidad. El caso español, a diferencia de Suiza o Bélgica, donde la voluntad pluralista marca dinámicas comunicativas plurales, es paradigmático porque continúa reproduciendo las formas de presentación de una España uniforme. Esto no es solamente una sensación cotidiana de aquellos que no se sienten representados por RTVE, sino que ha sido analizado por los académicos y ha impulsado la mayoría de las políticas de 
construcción de los medios de comunicación autonómicos alternativos al discurso estatal (Bustamante, 1985). Con el regreso de la democracia, la transición comunicativa fue finalmente continuista y no logró impulsar una perspectiva pluralista, razón por la cual actualmente continuamos con un modelo televisivo de la España castiza y mononacional, como bien indica en sus investigaciones Àlvar Peris Blanes (2008: 12).

Hablamos entonces de socialización, interiorización de la nación, nation building, vida cotidiana y medios, pero ¿cómo se da la correlación entre la nación y los espacios de comunicación? Como explica Josep Gifreu, cada nación necesita que las instituciones mediáticas colaboren en validarla y reproducirla simbólicamente. En los casos en que esto no sucede, hay disputas de los nacionalismos por controlar el espacio de comunicación, porque ambos espacios se superponen. En este sentido, decimos que un espacio nacional se construye sobre la base de una comunidad de cultura con unos referentes compartidos, instituciones políticas representativas o voluntad de tenerlas, con instituciones comunicativas en lengua propia que lleguen a todo el territorio (Gifreu, 1987: 54). Así, el objetivo central del espacio nacional de comunicación catalán es hacer del catalán lengua vehicular y hegemónica, recuperar la lengua y recuperar la nación, sobre la base de la comunicación. Como se ve aquí, la relación entre instituciones y construcción de comunidad y entre relaciones políticas y culturales es fundamental porque quien logra hacer que su visión de la nación sea hegemónica es quien puede dictaminar su forma y sus contenidos. Cuando los autores hablan de "reconstrucción nacional" hablan del proceso por el cual se pueden recuperar los "perfiles culturales propios", en este caso reprimidos por el estado español en su etapa franquista, sobre la base de crear espacios de comunicación donde se desarrollen las "aspiraciones políticas nacionales" y reproduzca la idea de nación compartida (Cardús, 2008: 29).

Para Josep Gifreu, El meu país: narratives i combats per la identitat de 2001, el Estado es el policía de la nación, es el centro vertebrador de la gran narrativa que sostiene la identidad nacional reproducida por el estado. Las narrativas sobre la nación son parte de la cultura nacional, no son esencias, sino discursos que van cambiando constantemente; al igual que el sentimiento nacional, que es un habitus internalizado, como diría Bourdieu, a través de un proceso de socialización que produce esquemas de percepción, ideas comunes, actitudes emocionales compartidas intersubjetivamente y predisposiciones a la acción. En el caso catalán, con un índice tan importante de inmigración, los medios de comunicación y la construcción de un espacio propio es fundamental para socializar en la nación a todos aquellos que llegan a Cataluña habiendo ya superado las instancias educativas obligatorias. Sin la posibilidad de asimilar tanta cantidad porcentual de recién llegados, con grandes 
dificultades para conocer catalanes y sin la existencia de beneficios directos de la integración, los medios masivos son unas de las pocas fuentes que los inmigrantes tienen de contacto con la cultura catalana (Gifreu, 2001: 99).

Tenemos entonces planteado el problema: en un territorio pueden coexistir diferentes nacionalidades, diferentes disputas entre nacionalismos por ser hegemónicos y diferentes espacios con dinámicas nacionales en conflicto por consolidar estas nacionalidades. Pero hay otro problema, los medios pueden ser públicos y privados y, según esta realidad, pueden colaborar con las lógicas de una o de otra nación. Como indica Peris Blanes, en el caso catalán existen medios públicos (RTVE) y privados (Telecinco, etc.) que apoyan el espacio español y reproducen la nación española y únicamente medios públicos autonómicos (TV3 y Cataluña Radio) que apoyan el espacio catalán. En el caso del espacio español de comunicación en Cataluña, los medios públicos y privados colaboran en un objetivo común, reinventar y reproducir la nación, lo que no sucede en el caso catalán. Además, tenemos que tener en cuenta que el espacio español se reproduce dentro del territorio catalán, porque forma parte del Estado, pero no a la inversa, desconociendo la variedad pluricultural del Estado.

En las televisiones públicas y privadas, prevalece casi siempre la unidad y homogeneidad de la nación española, fundamentalmente porque nunca se pone en cuestión. (...) Las televisiones españolas, públicas y privadas, saben perfectamente a quién se dirigen (la nación) y en qué lengua deben hacerlo (la castellana). Asimismo, conocen cuáles son sus principales instituciones, (monarquía, Cortes Generales, religión católica); las fiestas 'nacionales', a las que dedican una programación especial; y cuáles son los elementos que caracterizan la cultura nacional española, como los toros y el flamenco (Peris Blanes, 2008: 13).

Los medios masivos reproducen la nación como una obviedad, en los debates, en los mapas, en los temas seleccionados, en el clima. Los medios desarrollan y sostienen la nación porque cumplen una función narrativa rutinaria, de fundar marcos y de mediación, se encuentran entre nosotros y nuestra realidad, y están presentes en las formas en que nos informamos y decidimos sobre nuestra realidad. Si bien es cierto que analíticamente hay que considerar los medios como actores autónomos, también hay que pensarlos como instrumentos mediadores (producen objetos desde un punto de vista, ponen en circulación visiones del mundo y de cómo va el mundo, ponen orden en el caos aparente del mundo, valoran y toman posición) y como objetos de negociación estratégico (como factor de poder en las negociaciones de la opinión pública son disputados por las diferentes perspectivas). 


\subsection{Contexto histórico}

"No hay autonomía comunicativa sin autonomía política" (de Moragas, 1988: 12)

Con el regreso de la democracia en España, las instituciones catalanas comienzan a prepararse para recuperar sus instituciones y la autonomía que se había perdido con la Guerra Civil. La recuperación política fue posible gracias a las negociaciones para la restauración de la Generalitat, la firma de la Constitución española de 1978 y del Estatuto de Autonomía de Cataluña en 1979. A partir de esta nueva Constitución y de los pactos de gobernabilidad firmados por el partido catalán mayoritario, CiU, con ambos partidos españoles, las competencias catalanas se fueron recuperando poco a poco.

La recuperación cultural en Cataluña fue paradójicamente más simple, ya que existían instituciones de la sociedad civil que habían continuado la tarea de reproducción nacional y cultural durante el franquismo, si bien con un alcance limitado. Aunque el panorama parece mejor en el aspecto cultural que en el político, no hay consenso entre los analistas en que la cultura catalana se encuentre a salvo. Por esta razón, diferentes académicos, políticos y sectores de la sociedad civil concuerdan en que hay que crear políticas institucionales de producción cultural. El inicio de las transmisiones de la cadena autonómica (la Corporación Catalana de Radio y Televisión CCRTV) y de la escolarización en catalán de modelo único parece un éxito a medias porque muchos indican que no se ha logrado construir un espacio catalán de comunicación ni un uso público mayoritario del catalán como lengua vehicular.

Como señala Miquel de Moragas (1988), a partir de esta preocupación es que surge la reflexión sobre la necesidad de construir y potenciar un espacio nacional propio, proyecto llevado adelante por un grupo de la Escuela Catalana de Comunicación y apoyado en las reflexiones de la UNESCO. Durante los años sesenta, esta institución internacional recomendaba a los estados importar tecnologías de comunicación para poder desarrollarse, pero luego de una serie de investigaciones latinoamericanas que indicaban que estas políticas generaban más dependencia y no menos, se comienzan a recomendar formas de desarrollo independiente, conocidas como el Nuevo orden mundial de la comunicación. Este es el contexto también del debate intervencionismo estatal o libre comercio, la propuesta del eje socialista y de los países del Estado de Bienestar, como Suecia, frente a los neoliberalistas, principalmente Estados Unidos e Inglaterra. Los nuevos proyectos, que iban por la primera vía, buscaban expandir la comunicación y la alfabetización para fomentar el conocimiento, la producción y la democracia. "A la idea aceptada tradicionalmente de que no es posible la democracia sin circulación pluralista de información, le sigue ahora la evidencia de la necesidad de 
una política más amplia para dinamizar los programas culturales, sociales, económicos y políticos" (de Moragas, 1988: 39).

Este nuevo orden se encontraba directamente relacionado con las Políticas Nacionales de Comunicación, producto del debate sobre el Informe MacBride (1980), y el del apoyo de la UNESCO a los Países No Alineados en su pugna contra el neoliberalismo occidental que, al proponer la libre circulación de bienes culturales, beneficiaba a las grandes multinacionales en detrimento de las especificidades y necesidades de las naciones en vías de desarrollo. Esta disputa, que termina siendo un problema diplomático internacional, concluyó con la retirada de los Estados Unidos y de Gran Bretaña de la UNESCO por la presión de sus grandes periódicos y grupos de poder. La UNESCO comienza entonces a impulsar propuestas de neutralización de los desequilibrios comunicativos, por ejemplo vía la creación de agencias de noticias nacionales. La Conferencia Mundial sobre Políticas Culturales organizada en México (Mondiacult-1982), estaba destinada principalmente a fortalecer las políticas culturales en un contexto de imperialismo cultural: "limitar las influencias que pueden conducir al dominio cultural es hoy una tarea urgente" (Gifreu, 1986a: 251). Para 1986, y sobre esta base, en la Conferencia de Ministros europeos en Viena se propone definitivamente un modelo europeo de solidaridad para la defensa de los espacios nacionales de comunicación.

Retomaremos el pasado de Cataluña para entender un poco mejor por qué el regreso de la democracia es fundamental para el desarrollo de esta teoría. Luego de la Renaixença (Risques, 1999), se logra a finales del siglo XIX organizar una cierta prensa en catalán, con el Diari Català o la propia revista Renaixença, pero los grandes diarios como el Diario de Barcelona o La Vanguardia son en castellano. Podemos decir que hay una escisión entre la cultura de élite, de habla castellana, y la cultura más popular, de habla catalana. Luego de la Guerra Civil y con el franquismo, los sectores populares urbanos se alejan del catalán y será una apuesta política, antifranquista, recuperar poco a poco la lengua (Tresserras, 2010: 13). Durante este periodo dictatorial de cuarenta años, la televisión en castellano fue el elemento más importante de nacionalización y de difusión cultural del franquismo. Como observa Joan Manuel Tresserras: "iNosotros simplemente no podíamos aceptarlo! Al final de la dictadura no había prensa escrita en catalán, no había radio en catalán, no había televisión en catalán, etc. Estábamos cercanos a la nada" (Castelló, 2012: 93).

Recién cinco años después de iniciada la transición política, en 1982, se comienzan a sentir en Cataluña los efectos de la transición comunicativa y de la modernización definitiva de los medios de comunicación que permitirán su democratización (Guillamet, 1996: 233). Esto se produce gracias al apoyo de varios actores: la iniciativa privada con objetivos político-culturales (principalmente catalanista), las empresas 
mediáticas (a favor de la democracia pero en lengua castellana), las iniciativas locales y populares y la CCRTV (la acción comunicativa de la Generalitat de Cataluña desde 1984). Dentro del contexto del final de la dictadura y sobre las bases del proyecto MacBride, cierto sector de los académicos catalanes de la comunicación comienzan a llevar adelante una propuesta teórica, con incidencias políticas, de análisis del sistema comunicativo para poder construir el espacio catalán de comunicación.

\subsection{La teoría, el concepto y la acción}

La formulación de esta idea como una estrategia de reconstrucción nacional fue fruto fundamentalmente de una línea de investigación, no de un proyecto político (Gifreu, 2007: 4).

Como propone Jordi Berrio en su análisis de la Escuela catalana de comunicación, es con la transición que se dan las condiciones propicias dentro del campo político y académico para llevar adelante sus propuestas. Durante este periodo desarrollaron sus tareas Miquel de Moragas, Josep Gifreu y Joan Manuel Tresserras. Estos fueron interesándose por diferentes temas, pero el de las estructuras y políticas de cultura y comunicación fue siempre el más trabajado. Gracias a la tarea desarrollada por ellos y por sus equipos de investigación se ha llegado a una buena descripción del sistema comunicativo, si bien no se ha avanzado lo suficiente en la implementación de medidas políticas concretas (Berrio, 1998: 48).

El mundo académico, la sociedad civil y las instituciones catalanas se fueron coordinando, principalmente a partir del Congreso de Cultura Catalana de 1978, haciendo propuestas públicas y organizando centros como el $\mathrm{ClC}$, el Centro de Investigaciones en Comunicación, o el CAC, el Consejo Audiovisual de Cataluña, etc. En 1979 la situación de la lengua catalana preocupa y Josep Gifreu hace un análisis de la prensa en Cataluña donde indica que el $94 \%$ es en castellano. Al mismo tiempo que urge a revertir estos índices, denuncia que las propuestas del nuevo Estatuto de Autonomía no lo contemplan y que la Generalitat debería desarrollar "una política proteccionista para toda la prensa en catalán" (Gifreu, 1979: 27). Este proceso debería permitir pasar $6 \%$, en 1978 , al $25 \%$, el porcentaje correspondiente a 1936 , antes de la Guerra Civil y del Franquismo.

El proyecto de análisis mediático desde este paradigma da sus frutos en el libro colectivo Comunicació, llengua i cultura a Catalunya: horitzó 1990. Prospectiva sobre la transformació del sistema de comunicació a Catalunya ila seva incidència en la Ilengua i la cultura dirigido por Josep Gifreu (1986a), el análisis más exhaustivo del sistema mediático en los Países Catalanes que se haya emprendido hasta ahora. Ya 
desde la introducción los autores dejan en claro tres aspectos: por un lado la nueva realidad de la penetración gradual pero irresistible de las nuevas tecnologías en el tejido social; los problemas técnicos y culturales derivados de los procesos globalizadores neoliberales; y los problemas propios de Cataluña provenientes de ser una nación sin Estado ${ }^{3}$ y con un mercado integrado al español.

Como consecuencia, hay que entender que puede resultar altamente temerario para el futuro de la reconstrucción nacional no disponer de unos mecanismos políticos adecuados para el control de la nueva dinámica cultural, impuesta por las industrias transnacionales de la cultura y de la información (Gifreu, 1986: 255).

A partir de aquí se hacen una serie de propuestas de actuación, lo que demuestra no solamente una voluntad teórica-analítica, sino también política, de acción. En relación con la identidad nacional indican que hay que: 1 . garantizar el uso del catalán; 2. valorar la producción propia y no la importación; 3. trabajar sobre la apropiación y no sobre la difusión de la cultura ajena; 4. apoyar las prácticas colectivas sobre el consumo individual; 5 . promocionar un espacio compartido y de colaboración para potenciar la identidad de los Países Catalanes. Este concepto, que venía del antifranquismo, es fundamental para entender la propuesta del espacio catalán de comunicación porque este incluía a todos los territorios de habla catalana y no únicamente a la Comunidad Autónoma de Cataluña. En relación con la necesidad de construir una democracia cultural, señalan que hay que asegurar: 1 . la diversidad; 2 . la disponibilidad; 3. la participación activa (acceso, participación y autogestión) y; 4 . la articulación entre culturas (élite, masas y popular). En última instancia indican que no es cierto que la política no pueda controlar la tecnología, pero hay que estar decididos y esta no ha sido esta la actitud de la Generalitat (Gifreu, 1986a: 245).

En un libro del año 1989, Comunicació i reconstrucció nacional, Josep Gifreu, que ya había reflexionado sobre las lógicas internacionales del nuevo orden mundial y había hecho un análisis del sistema comunicativo, decide reflexionar sobre la historia del siglo de las dictaduras españolas en clave comunicacional. Propone así pensar el franquismo en dos etapas: la primera entre 1937 y 1966, cuando la dictadura va anexionando los diversos espacios del territorio español para instituir un único espacio, así es que se implementa la Ley de prensa de 1938 y la censura (ver Guillamet, 1996: 57), se crea Radio Nacional de España (donde se excluyen todas las lenguas que no sean el español), la Escuela Oficial de Periodismo en 1941 y el Ministerio de Información y Turismo en 1951; y una segunda etapa entre 1966 y 1975, donde la crisis

\footnotetext{
${ }^{3}$ El concepto de nación sin Estado es utilizado extensamente en la bibliografía sobre comunicación y sobre nacionalismo para describir la situación catalana, un territorio que se considera una nación pero que no tiene un Estado como marco político. Para más información ver Guibernau Montserrat 1999.
} 
del régimen promueve una serie de medidas, como la nueva Ley de Prensa de 1966, que buscaba actualizar los postulados franquistas a la nueva realidad, pero, poco a poco, comienzan a resurgir circuitos de difusión sobre la base de los antiguos espacios de comunicación regionales. Guillamet considera que a partir de 1966 comienzan once años de libertad vigilada donde, si bien se había superado ya la censura como institución, el Estado y los propios redactores seguían actuando como censores y autocensores (Guillamet, 1996: 89 y 111). Con el postfranquismo se quieren restituir estos espacios nacionales pero no se toca el modelo heredado, lo que termina generando que la transición consolide democráticamente la conciencia nacional creada por el franquismo.

Finalmente, para Gifreu (2007: 3) hay cuatro momentos fundacionales de esta reflexión: 1. en 1986 da la conferencia inaugural de las II Reflexions Crítiques de la Cultura Catalana, donde propone por primera vez la necesidad de promover el espacio comunicacional catalán; 2. en 1991, cuando el Presidente de la Generalitat, Jordi Pujol, presenta en público el informe Construir l'espai català de comunicació, elaborado por él con un grupo de colabores de todos los Países Catalanes; 3. en 1999, cuando el Parlamento de Cataluña aprueba por unanimidad la resolución 3/VI donde se proponen las bases normativas para la promoción del espacio comunicativo; 4 . en 2005, cuando el mismo Parlamento aprueba la nueva Ley de la Comunicación Audiovisual.

El informe de 1991 deja entrever que el prometido espacio europeo de comunicación no existe, probablemente porque Europa no tiene un proyecto político ni cultural conjunto. Una vez cerrada la posibilidad de un diálogo entre las regiones europeas, el camino pasa por establecer un Pacto Cultural y de Comunicación de los Países Catalanes, la creación de una agenda de noticias, una cadena de TV y un diario interregional, además de estimular la investigación sobre la base de informes periódicos sobre "el estado de la catalanidad". Cuatro años después, y con el ingreso del Partido Popular en la presidencia de la Comunidad Valenciana, comienza lo que la mayoría de los análisis cifran como el final del proyecto de los Países Catalanes. Los problemas serán constantes a la hora de intentar realizar proyectos pancatalanes que reunifiquen la nacionalidad catalana distribuida entre cuatro comunidades autónomas separadas, no solo administrativamente sino también ideológicamente.

En los noventa, el panorama catalán cambia significativamente por el ingreso de las nuevas tecnologías, el crecimiento del poder adquisitivo y de la inmigración. Esto modifica la problemática propuesta, pero no cambia la perspectiva, es más, se acentúa el escepticismo y el sentimiento de que se ha dejado pasar una gran oportunidad. En el año 2001 Josep Gifreu publica el ensayo El Meu país: narratives i combats per la identitat, "con la sensación de que el país se está acabando". Las estadísticas indican 
que en el año 2000 el 70\% del consumo televisivo en Cataluña es producido en España y en español, lo que puede empeorar con la liberalización de las nuevas cadenas de TV y la TDT, un golpe duro producido por la incapacidad de la Generalitat de controlar estas nuevas realidades tecnológicas. Para este contexto propone una serie de hipótesis de trabajo: 1 . el ecosistema televisivo presente en Cataluña tiende a la minorización del catalán; 2 . los altos rendimientos de las audiencias catalanas son un factor productivo para la industria española, pero no para la catalana; 3 . la comunicación política tiende a considerar a la política catalana como subsidiaria de la política española; y 4 . las cadenas españolas de televisión crean y distribuyen una imagen distorsionada de la identidad catalana (Gifreu, 2001: 151).

Finalmente, Gifreu en su penúltimo libro, La Pell de la diferència: comunicació, Ilengua i cultura des de l'espai català de 2005, propone otro diagnóstico esclarecedor: 1 . no ha habido un análisis claro de la crisis ni se han entendido las nuevas formas de dependencia de la cultura catalana; 2 . se ha entendido la cultura como una cuestión administrativa de gestión del patrimonio; 3 . han faltado los mecanismos políticos para llevar adelante una política cultural que desarrolle las estrategias centrales de la reconstrucción nacional. La cuestión de fondo es:

¿Qué capacidad de control tiene Cataluña sobre la dinámica cultural impuesta, de un lado, por sus relaciones de dependencia del Estado español y de la cultura castellana y, del otro, por las industrias transnacionales de la cultura de la información? (Gifreu, 2005: 38).

\subsection{Balance de una propuesta}

Tenemos derecho legítimo a intervenir sobre un mercado heredado y que fue configurado por unas políticas impuestas de los que habían ganado una guerra (Tresserras, 2010: 20).

Hasta aquí hemos recuperado una propuesta teórica-política histórica y presente, la forma en que la escuela catalana de comunicación ha participado de la reconstrucción nacional catalana. Esta perspectiva de análisis, que busca crear propuestas concretas para el avance cultural, ha ido de la mano de la historia de la nación y del Estado y de esta relación han nacido sus posibilidades y sus limitaciones. En este apartado hacemos un balance de la situación y de los temas que fueron centrales en el proceso de construcción del espacio catalán durante los últimos treinta años.

Joan Manuel Tresserras (2010) apunta que no se ha logrado que los productos catalanes tengan el valor agregado de ser expresión de catalanidad, de ser diferentes y 
más próximos que los españoles; no se ha logrado que el público los sienta cercanos como un factor de complicidad. El esfuerzo debería estar puesto acá, en producir con calidad y, al mismo tiempo, ganar autoestima para lograr que el negocio de los productos catalanes funcione económicamente. En este sentido, el fracaso más grande ha sido la imposibilidad de crear un mercado normalizado con su dinámica propia, aunque, paradójicamente, la escolarización y el espacio catalán se hayan desarrollado más o menos efectivamente. Son las instituciones públicas catalanas las que tienen que asegurarse de crear el público y de que les lleguen los productos, la empresa privada no se encargará de crearlo y seguirá haciendo el negocio donde más beneficios obtengan.

El balance propuesto por Josep Gifreu (2007) comienza actualizando la definición del espacio catalán que se ha propuesto como una estrategia general de acción no partidista, donde se ha de implicar a todos los partidos, no limitada a un área del dominio lingüístico y destinado a la reconstrucción cultural. En relación con los puntos fuertes en los Países Catalanes hay que valorar las nuevas fortalezas de las políticas autonómicas regionales y el desarrollo de corporaciones públicas de radio y televisión regionales (RTVV Radio y Televisión Valenciana y IB3 Radiotelevisión de las Islas Baleares), la existencia de redes de colaboración entre entidades culturales de la sociedad civil (Xarxa Vives, la Federació Llull, etc.) y el despliegue de algunas iniciativas empresariales en el campo de la comunicación en catalán (El Temps, Vilaweb y el Baròmetre de la Comunicació).

En relación con los puntos fuertes hay que tener en cuenta el reconocimiento social de las políticas lingüísticas de discriminación positiva para el catalán, principalmente en los medios masivos, la tarea indiscutible en generar consenso social de la Corporación Catalana de Medios Audiovisuales (la CCMA, heredera de la CCRTV), la capacidad creativa de las industrias culturales catalanas, una dinámica activa entre las propuestas locales y comarcales y los nuevos medios digitales territoriales. En relación con los puntos fuertes del espacio exterior, hay que valorar la creciente presencia internacional del espacio cultural catalán, la importancia del .cat y del lugar del catalán en internet, al igual que de la marca Cataluña (por ejemplo con el éxito de la Feria del libro de Frankfurt de 2007) y de los referentes culturales considerados catalanes, Antoni Gaudí, Pau Casals, Salvador Dalí, Joan Miró, etc.

Los puntos débiles del espacio cultural en los Países Catalanes son la extrema politización de la lengua y de la cultura catalana, la falta de un estatuto de la lengua catalana en el Estado español y en la Unión Europea, las dudas sobre su unidad o sobre los territorios de referencia, falta de un mercado común protegido y de unas industrias culturales fuertes, la progresiva españolización de los contenidos y referentes de los grandes medios así como la inexistencia de organizaciones interterritoriales de 
producción y distribución. Los puntos débiles en Cataluña tienen que ver con que ninguno de los grandes grupos mediáticos han apostado por el espacio catalán, orientándose al mercado español y en español; si bien existe el CAC (Consejo del Audiovisual de Cataluña), este no tiene autoridad sobre el $80 \%$ de los productos que se consumen en Cataluña y, finalmente, la peligrosa penetración de grupos privados españoles en todos los sectores. En relación con los puntos débiles del exterior, hay que admitir una falta de reconocimiento del Estado español, y por lo tanto de Europa, de la excepción cultural catalana.

En relación con los puntos negros de los Países Catalanes, Gifreu reconoce la inexistencia de medios que cubran informativa y culturalmente las necesidades de los ciudadanos del territorio común, así como la falta de un pacto por la cultura y la comunicación entre las regiones catalanoparlantes y la expansión inexorable del espacio español sobre el catalán. Y para terminar, los puntos negros hacia el exterior provienen de la falta de reconocimiento del catalán como lengua oficial en la Unión Europea, la falta de visibilidad global y la imposibilidad del acceso a los programas de promoción, la falta de un Estado protector y de un canal internacional de radio y televisión representativos. Como reconocía en su balance de 2008 Salvador Cardús:

La comunidad lingüística catalana, como es sabido, es superior a la del territorio de la Comunidad Autónoma de Cataluña, ya que incluye las comunidades autónomas valencianas - el País Valenciano - y de las Islas Baleares, así como una parte del sur del territorio francés, incluyendo un total aproximado de 12 millones de personas. TV3 y Cataluña Radio han llegado a la totalidad del espacio de comunicación, pero los obstáculos políticos - en un tiempo que se dice "sin fronteras"- han sido constantes y en muchas ocasiones insalvables (Cardús, 2008: 43).

Como ha quedado en claro luego de este recorrido teórico e histórico, la mayoría de los autores trabajados consideran que, por lo menos, el proceso de construcción del espacio nacional catalán fue insuficiente. Lo que sí se ha logrado es un reconocimiento, no formal, de la condición de nación de Cataluña y de sus características culturales diferenciadas del resto de España. Si bien este reconocimiento social, que no legal, ha ayudado mucho a la dignificación de la cultura, se ha dado un proceso inverso de politización y de negación que ha minado las posibilidades de coordinar políticas comunes entre las cuatro comunidades que conforman los Países Catalanes. En este sentido, el proyecto conjunto se ha estancado y los cambios tecnológicos han ido generando nuevos problemas que han terminado reduciendo los beneficios que la construcción del espacio catalán había conseguido. 
Es unánime la crítica de la mayoría de los intelectuales al desinterés de los partidos políticos sobre este tema, la reconstrucción nacional ha sido en gran parte fruto de la participación de la sociedad civil. Aunque se ha avanzado mucho, existen muchos más catalanoparlantes que hace treinta años, hay una gran presencia en la red y se han construido espacios interesantes de producción y distribución de la cultura catalana, la opinión generalizada de los expertos es que hay mucho por hacer (Gifreu, 2008: 8). Si miramos hacia el pasado, veremos que la Europa de las naciones no se ha desarrollado; al no intervenir de manera conjunta en la educación, las culturas y las lenguas, Europa ha perdido su poder de crear un futuro mejor para sus naciones sin Estado.

Finalmente, Gifreu en su último informe L'acadèmia i les polítiques nacionals de comunicació a Catalunya: ideació, fulgor i declivi d'una escola de 2012 indica que nos encontramos en una etapa de declive de esta escuela. Para nosotros lo que está sucediendo no es un declive, sino un proceso de cambio que, al ser tan extremo, no reconoce su pasado, pero que es continuista. No podemos hablar de decadencia, pero sí de recambio generacional, cuando los intelectuales que comenzaron a defender este proceso en la transición política están dejando lugar a una nueva generación de jóvenes investigadores. Hablar de declive es muy complicado cuando los historiadores de la comunicación (Berrio, 1998) indican que los estudios sobre el sistema comunicacional catalán siguen creciendo y más investigadores que nunca trabajan para entender la realidad. Tampoco podemos hablar de decadencia cuando reconocemos la consolidación, de la Corporación de Radio y Televisión de Cataluña como eje vertebrador de las organizaciones mediáticas nacionales y cuando vemos la innegable importancia de la web 2.0. Más que decadencia, habría que hablar de recambio, de un nuevo contexto político, de nuevos actores académicos y políticos y de nuevos medios, de nuevas formas de participar de la comunidad catalana, más allá de las leyes y de las fronteras.

El recorrido nacional no es ajeno a su cultura y a sus medios de comunicación, por eso al preguntarle a Gifreu en una entrevista sobre los posibles escenarios del futuro, el horizonte del día después de un proceso de independencia de Cataluña de España, se muestra optimista, si bien cauteloso. En el hipotético caso de que el referéndum fuese negativo y no se produjese la independencia, Gifreu considera que las estructuras construidas hasta este momento son poderosas y están bien consolidadas, que no hay peligros frente a un futuro conflicto. En el caso contrario, con el horizonte de una transición nacional, Gifreu nos recuerda dos aspectos importantes: por un lado, que la independencia se produciría por parte de un territorio, si bien no menor, de los Países Catalanes que incluso generaría una nueva distancia frente a los otros territorios históricos de habla catalana y; por otro lado, indica que habría que redactar una 
Constitución catalana, construir nuevas estructuras estatales de comunicación y renegociar el $85 \%$ de los productos comunicativos españoles que se consumen en Cataluña sobre la base de acuerdos de cooperación y respetando la diversidad interna del país. Según Gifreu, estos son los dos frentes abiertos a la hora de pensar un futuro nacional y los espacios donde más disputas podrían plantearse.

Mucho se ha hecho y el futuro parece prometedor pero, por otro lado, la sensación es de fragilidad, que lo avanzado es insuficiente y queda siempre la posición de defensa. Es posible que esta sea la postura de aquellos intelectuales que provienen de la Transición y que el lugar de los nuevos académicos sea adelantarse al futuro haciéndolo posible, generando las condiciones de posibilidad para seguir avanzando en un proyecto colectivo catalán y pancatalán con objetivos nacionales.

\section{Conclusiones}

El nacionalismo, la idea de nación (y las estrategias para hacerla cotidiana) y los medios de comunicación son tres aspectos que no suelen relacionarse en las reflexiones académicas. Es por esta razón que para este artículo recurrimos a la historia y a la teoría para ver los puntos de contacto y convergencia entre la recuperación de la nación catalana en la transición y en la democracia y la construcción de un espacio nacional de comunicación en Cataluña. Un recorrido de 36 años entre políticas públicas y proyectos académicos que buscaban crear las instituciones necesarias para reproducir la idea de que Cataluña no era únicamente una región, sino una nación.

Luego de la muerte de Francisco Franco se marca la fecha de 1978 como clave para la transición cultural catalana gracias al Congreso de Cultura Catalana, al Nuevo Orden Mundial de la Comunicación apoyado por la UNESCO y a los nuevos espacios académicos que se iban creando. Este mismo año, la transición política saca adelante la nueva Constitución española y, al año siguiente, el Estatuto de Autonomía de Cataluña. Estaban ya sentadas las bases para superar el modelo comunicativo franquista de cultura única, para recuperar la pluralidad regional y nacional de una España democrática, la España de las Autonomías. La situación en que se encuentran los medios de comunicación catalanes al inicio de la década de los 80 preocupa a Miquel de Moragas, a Joan Manuel Tresserras y a Josep Gifreu, quien constata que el $94 \%$ de la prensa que se consume en Cataluña es en castellano.

El proyecto de crear un espacio nacional catalán de comunicación busca entonces que el catalán se convierta en lengua vehicular (y la cultura catalana en cultura compartida) 
de los medios de comunicación y de las industrias culturales que se consumen, no solamente en Cataluña, sino en los Países Catalanes. Este aspecto del proyecto, el territorial, fue el que menos éxito tuvo por las diferencias entre el gobierno catalán de CiU y el de Valencia y de las Islas Baleares, del Partido Popular. Las disputas políticas son también índice de las disputas entre concepciones nacionales diferentes, conflictos sobre la manera de concebir la nación catalana y española. La colaboración entre medios públicos y privados en la reproducción de esta idea de nación fue también poco beneficiosa para Cataluña, donde los medios privados españoles reproducen la misma idea monocultural de España que los medios estatales.

Finalmente, si bien el avance es considerable (para el año 2000 el 30\% del consumo televisivo era en catalán) la capacidad normativa de la Generalitat no ha mejorado ni las políticas compensatorias son suficientes. El tardío apoyo institucional a los medios tradicionales (positivo para la televisión y la radio y escaso para la prensa escrita) se suma a nuevos problemas, propios de las nuevas tecnologías de la comunicación y de la información: los nuevos medios, los social media, internet y las redes sociales. Los investigadores de la academia catalana han denunciado constantemente la falta de apoyo político a sus propuestas, la incapacidad política de entender la relevancia de las medidas propuestas. Con la nueva situación política catalana que se generó luego de las elecciones anticipadas de 2012 y con el proceso independentista, se abren nuevas posibilidades para reflotar el proyecto del espacio nacional de comunicación, nuevas posibilidades pero también nuevos retos.

\section{Referencias bibliográficas}

AMEZAGA, J. (2004): "Televisión por satélite en el mundo: nuevos retos para viejas lenguas", en: Portal de la Comunicación, Fórum Barcelona, 2004. Disponible en Internet

(06-09-2014): http://www.portalcomunicacion.com/dialeg/paper/pdf/16_amezaga.pdf

BERRIO, J. (1998): "La recerca sobre comunicació social a Catalunya: assaig de realització d'un panorama comprensiu", en: Anàlisi: Quaderns de comunicació $i$ cultura, no 22, p. 47-59.

BUSTAMANTE, E. (1985): "Políticas de comunicación: un reto actual", en de Moragas, M., Sociología de la comunicación de masas, Barcelona, Gustavo Gili, vol. 4, p. 125-143. 
CARDÚS, S. (2008): "Televisió i identitat nacional. Esplendor i declivi de la televisió autonòmica catalana" en VIA06 Valors, Idees, Actituds, Barcelona, p. 36-52.

CASTELLÓ, E. (2007): Sèries de ficció i construcció nacional: imaginant una Catalunya televisiva, Tarragona, $\mathrm{A}+\mathrm{C}$.

- (2008): Identidades mediáticas: introducción a las teorías, métodos y casos, Barcelona, UOC.

- (ed.) (2012): La mediatización del conflicto político: discursos y narrativas en el contexto español, Barcelona, Laertes.

COROMINAS, M. (1994): “Aproximació catalana a la comunicació", en Berrio, J. (dir.), Un Segle de recerca sobre comunicació a Catalunya, Bellaterra, UAB, p. 110-127.

DE MORAGAS, M. (ed.) (1986): Sociología de la comunicación de masas, Barcelona, Gustavo Gili.

- (1988): Espais de comunicació: experiències i perspectives a Catalunya, Barcelona, Edicions 62.

- (1990): Teorías de la comunicación: investigaciones sobre medios en América y Europa, Barcelona, Gustavo Gili.

DE MORAGAS, M., FERNÁNDEZ, I. (eds.) (2008): Communication and cultural policies in Europe, Barcelona, INCOM-UAB.

FERNÁNDEZ, J. A. (1995): "Becoming Normal: Cultural Production and Cultural Policy in Catalonia", en Graham, E, Labanyi, J., Spanish cultural studies: an introduction, Oxford, University Press, p. 343-346.

GIFREU, J. (1979): “Premsa a Catalunya: quasi tota en castellà", en Revista L'Hora de Catalunya, no 36, p. 25-28.

- (dir.) (1986a): Comunicació, llengua i cultura a Catalunya: horitzó 1990, Barcelona, Institut d'Estudis Catalans.

- (1986b): El debate internacional de la comunicación, Barcelona, Ariel.

- (dir.) (1987): Segones reflexions crítiques sobre la cultura catalana, Barcelona, Departament de Cultura de la Generalitat de Catalunya.

- (1990): “España, un espacio de comunicación en crisis", en Revista Telos, no 23, Madrid, p.10-12.

- (1989): Comunicació i reconstrucció nacional, Barcelona, Pòrtic. 
- (dir.) (1991): Construir l'espai català de comunicación, Barcelona, Generalitat de Catalunya y Centre d'Investigació de la Comunicació.

- (2001): El meu país: narratives i combats per la identitat, Lleida, Pagès.

- (2003): La potenciació de l'espai cultural i audiovisual català: estudi prospectiu i proposta de líneas d'actuació, Barcelona, Generalitat de Catalunya.

- (2005): La pell de la diferència: comunicació, llengua i cultura des de l'espai català, Barcelona, Pòrtic.

- (2007): “20 anys de l'espai Català de comunicación. Un objectiu encara possible?", en ESCACC, Documents 8, Barcelona. Disponible en Internet (06-092014):

http://culturaisocietatalsppcc.wikispaces.com/file/view/Tema+4.+Mitjans+de+co nmunicaci\%C3\%B3.+Conferencia_escacc.pdf

- (2012): “L'acadèmia i les polítiques nacionals de comunicació a Catalunya: ideació, fulgor i declivi d'una escola", en L'Informe de la comunicació a Catalunya 2011-2012. Disponible en Internet (06-09-2014): http://incom.uab.cat/informe/

GIORI, P. (2013): “Catalanisme cultural: reptes i possibilitats de construir una nació en democracia", en Revista Afers: fulls de recerca i pensament, n.76, Catarroja, p.807-824.

GUIBERNAU, M. (1999): Nacions sense Estat: nacionalisme i diversitat en l'era global, Barcelona, Columna.

GUILLAMET, J. (1996): Premsa, franquisme i autonomía. Crónica catalana de mig segle llarg (1939-1995), Barcelona, Flor del Vent.

INFORME MACBRIDE (1980): Voces múltiples, un solo mundo. Disponible en Internet (06-09-2014): http://unesdoc.unesco.org/images/0004/000400/040066sb.pdf

LO CASCIO, P. (2008): Nacionalisme i autogovern: Catalunya, 1980-2003, Catarroja, Afers.

PERIS BLANES, À (2008): “Identidad nacional, televisión y vida diaria. Perspectivas para un debate sobre el caso español", en MARíN, N., ENCARNA, M., GONZÁLEZ, C., Ayeres en discusión. Temas clave de Historia Contemporánea hoy, Murcia, Universidad de Murcia, p. 1-23.

RISQUES, M. (dir.) (1999): Història de la Catalunya contemporània, Barcelona, Pòrtic. 
ROMEU JOVER, X. (ed.) (1977): Debat sobre els Països Catalans: ponències $i$ comunicacions de les Jornades de Debat sobre els Països Catalans (octubre 1976), Barcelona, Curial.

TRESSERRAS, J. M. (2010): Polítiques de cultura i comunicació i construcció nacional, Barcelona, Generalitat de Catalunya.

VVAA. (1978): Congrés de Cultura Catalana, Resolucions 3, Barcelona, Curial. 